\title{
Guimarães Rosa und die Demokratie auf dem Weg
}

\author{
Herausforderungen des ZusammenLebensWissens in Brasilien
}

\section{Erster Schritt: Streiflichter des Guimarães Rosa auf Brasiliens Geschichte - von der letzten Station aus}

Am 16. November 1967, gut zweieinhalb Jahre nach Beginn der letzten Militärdiktatur in Brasilien, trat João Guimarães Rosa (1908-1967) seinen Sitz an der brasilianischen Literaturakademie an. Drei Tage später starb er 59-jährig an einem Herzinfarkt. ${ }^{1}$ In der Rede, die der neue «Unsterbliche» nach gut viereinhalb Jahren Wartezeit seit seiner Ernennung gehalten hatte, folgte er der Tradition des Hauses und lobte seinen Vorgänger João Neves da Fontoura (1887-1963) - und zwar genau am Datum von dessen 80. Geburtstag. Dieses runde Jubiläum sei der Grund für den späten Antritt der wichtigen Honorierung gewesen, erklärte Rosa am Ende seiner Rede. ${ }^{2}$ Die Verzögerung des Antritts um vier Jahre: eine aus heutiger Sicht etwas exzentrische, vielleicht deshalb aber umso bedeutendere Geste der Entsagung und der Würdigung eines Anderen zugleich.

Ehemaliger Bundesminister für Außenbeziehungen (1946 in der Regierung Dutra, dann wieder 1951-1953 in der zweiten, diesmal demokratisch gewählten Regierung Vargas) war Neves da Fontoura am 31. März 1963 gestorben, genau ein Jahr vor dem Militärputsch, der die besagte, 24 Jahre währende Militärdiktatur in-

\footnotetext{
1 Vgl hierzu Marcelo Marinho: João Guimarães Rosa, «autobiografia irracional» e crítica literária: veredas de oratura. In: Letras de Hoje 47/2 (2012), S. 186-193. Camila Moreira Cesar/Marcelo Marinho: A mídia e a construção de personagens de autoficção biográfica: uma leitura semântico-lexical de três notícias sobre a morte enigmática de João Guimarães Rosa. In: Letras de Hoje 52/2 (2017), S. 115-128. Marcelo Marinho/David Lopes da Silva: «Desenredo», de João Guimarães Rosa: prosoema, metapoesia, necrológio prévio ou «autobiografia irracional»? In: Remate de Males 39/2 (2019), S. 799-829. Die Autoren vertreten die Auffassung, dass der frühe Tod von Guimarães Rosa einer durch den Schriftsteller genau kalkulierten Inszenierung der eigenen Biographie - und des gewollten Ablebens - zurückzuführen wäre.

2 Die Antrittsrede («Discurso de posse») wird hier in der Fassung der Webseite der Academia Brasileira de Letras zitiert: http://www.academia.org.br/print/18/discurso-de-posse. Letzter Zugriff: 30.1.2018.
}

Ә Open Access. (C) 2020 Paulo Astor Soethe, publiziert von De Gruyter. (c) BY-NC-ND Dieses Werk ist lizenziert unter der Creative Commons Attribution-NonCommercial-NoDerivatives 4.0 Lizenz. https://doi.org/10.1515/9783110677713-002 
augurierte. Die Nachfolge des wichtigen Politikers aus Rio Grande do Sul trat Guimarães Rosa, der Diplomat aus Minas Gerais, mit den vier Jahren Verzögerung in repräsentativer Form an. Dies war kein rein protokollarischer Fall: Er und sein Vorgänger in der Akademie waren durch eine lange, intensive Zusammenarbeit im Außenministerium Brasiliens eng verbunden, Rosa hatte ja beide Mal beim Minister als dessen erster Staatssekretär gewirkt, und mit Recht hielt er seinen Vorgesetzten und guten Freund für eine der zentralsten Figuren in der brasilianischen Geschichte zwischen 1929 und 1954. Mit seinen beeindruckenden Reden, entschiedener Position als Demokrat und Republikaner, mit seiner Kunst, wichtige Bündnisse und Brüche in der politischen Szene aufzubauen und zu vermitteln, sammelte Fontoura im Laufe seines öffentlichen Lebens große Verdienste, zumal er gegenüber der umstrittenen, ihm aber sehr nahen Figur von Getúlio Vargas (1882-1954), große Autonomie beizubehalten wusste. Er nahm eine kritische Haltung ein und geriert daher mit dem mächtigsten Mann jener bewegten Jahre nicht selten in Konflikt. Auf die Eloquenz, Führungskapazität und Scharfsinnigkeit von Neves da Fontoura konnte selbst der in Frage gestellte Diktator nicht verzichten.

Denn schon vor Beginn der ersten Regierungszeit von Vargas zwischen 1930 und 1945, als mittelständische, zumeist fortschrittliche Kräfte die sogenannte Aliança Liberal (Liberale Allianz) zu bilden imstande waren, erklang in der Tribüne die Stimme von Neves da Fontoura als Verfechter der politischen Bewegung und führendem Wegweiser in die Überwindung der damals oligarchisch herrschenden Milchkaffee-Politik. ${ }^{3}$ In seiner Antrittsrede in der Academia Brasileira de Letras zelebriert Rosa enthusiastisch den 5. August 1929, den Tag, an dem der Abgeordnete Neves da Fontoura zur Ankündigung des neuen Bündnisabschlusses zwischen den Bundesstaaten Rio Grande do Sul, Minas Gerais und Paraíba mit Unterstützung fortschrittlicher Kräfte von São Paulo eine dreistündige Rede gehalten hat. Der große Romancier signalisierte somit, die politischen Ereignisse in Brasilien genau beobachtet zu haben, an deren Entfaltung João Neves da Fontoura mitwirkte. Dabei habe er manchmal die Position eines zelebrierten Protagonisten eingenommen, manchmal sei er wie ein von der Macht vernachlässigter, geächteter und verbannter Widersacher erschienen.

Beispielsweise war João Neves da Fontoura 1930 Vizegouverneur von Rio Grande do Sul, als Getúlio Vargas, der Gouverneur, mit Unterstützung mitstreitender Bundesstaaten durch einen Putsch auf das Amt des Präsidenten der Republik

3 Gemeint war damit der Zusammenschluss zwischen vermeintlichen Konkurrenten in der damaligen politischen Szene Brasiliens, die sich nach Absprache und abwechselnd an der Regierung die Macht und Kontrolle des Staates erhielten. Milch stand für die Großgrundbesitzer in der Viehzucht, vor allem im Minas Gerais und Rio Grande do Sul, Kaffee für die sogenannten Kaffeebarone im Bundesstaat São Paulo. 
aufstieg und somit das Ende der Ersten bzw. Alten Republik bestimmte. Doch die Macht in der südlichsten Provinz durfte auf Wunsch von Vargas ein anderer übernehmen, Osvaldo Aranha, und nicht Fontoura, gleichwohl er sich für den Aufstieg von Vargas sehr intensiv engagiert und in der erfolgreichen Bewegung eine führende Rolle übernommen hatte.

Zwei Jahre später, als 1932 demokratische Kräfte im Bundesstaat São Paulo zu einer Konstitutionellen Revolution aufriefen, um sich gegen die autoritäre Zentralisierung der politischen Macht durch Vargas zu wehren und die Wahl einer Verfassungsversammlung zu fordern, trat Neves da Fontoura in Rio Grande do Sul an der Seite der Aufständischen als einer der führenden Politiker in jenem oppositionellen Versuch der Demokratisierung, Stabilisierung und gerechteren Gewichtung der Machtverhältnisse im Land auf. Nach vier Monaten bewaffneten Konfliktes und dem militärischen Sieg der Zentralregierung über die Revolte wurden deren Anführer ins Exil verbannt, unter ihnen João Neves da Fontoura, der erst 1934, nachdem eine neue Verfassung verabschiedet worden war, aus Argentinien zurückkehrte. Schon im Folgejahr wurde er nochmals zum Abgeordneten gewählt und setzte so, auch während der Diktatur im brasilianischen Estado Novo (19371945), seine Laufbahn als wichtiger Akteur im gesellschaftlichen Leben Brasiliens fort. Ab Beginn der diktatorischen Zeit übernahm Fontoura bezeichnenderweise verschiedene Posten des diplomatischen Dienstes im Ausland.

João Guimarães Rosa lobt in Neves da Fontoura seine große Anpassungsfähigkeit und seinen Pragmatismus: er verzichte auf solide demokratische Prinzipien nicht («ele era, por constância e excelência, o democrata») aber er habe, weil er handlungsfähig bleiben wollte, Zugeständnisse machen müssen sowie Ambivalenz und Widersprüchlichkeit im Umgang mit der Macht nicht vermeiden können: «quem pensa no Brasil, e no povo do Brasil, vezes quantas rebeija pedras e santos.» ${ }^{4}$ Rosa behauptet es nicht ausdrücklich, doch er war sich dessen bewusst, dass die Basis für eine richtige, moderne Demokratie fehlte: aktive Bürgerinnen und Bürger de facto gab es wenig, denn die meisten Menschen waren rechtlos und dem Staat gegenüber völlig entfremdet. Die dünne herrschende Elite handelte oligarchisch und brutal, rechtsstaatliche Kategorien und ein demokratisches Verhalten waren für sie keine Selbstverständlichkeit. Politische Führer, die zugleich demokratische Denker waren und mit repräsentativen Gruppen ein Konzept für das Land vorlegen konnten und wollten, waren selten und in der herrschenden politischen Szene kaum unterzubringen. Sie wurden oft verbannt oder inhaftiert.

João Neves da Fontoura spielte in dieser Landschaft die seltene Rolle eines Pragmatikers, der als ziviler Jurist und politischer Denker demokratische Über-

4 Vgl. «Discurso de posse». 
zeugungen verbreitete, sie in Wirklichkeit möglichst umzusetzen versuchte und es dennoch präsent haben musste, dass unter den herrschenden Machtstrukturen sein Wort und sein Handeln eine eher erzieherische Funktion erfüllten.

Ein Beispiel, das in der Rede von Rosa zwar nicht ausführlich dargelegt wird, ${ }^{5}$ aber für die politische Rolle Fontouras kennzeichnend ist, sei an dieser Stelle kurz angeführt: In Fontouras Geburtsstadt Cachoeira do Sul, circa 100 Kilometer von Porto Alegre entfernt, war ihm als Intendant (d.h. Bürgermeister) gelungen, zwischen 1925 und 1929 die Gemeinde zu modernisieren und den Stadtkern zu urbanisieren. Unter seiner Regierung wurde ein Programm für Wasserversorgungsund Abwasserentsorgungsinfrastruktur durchgeführt, welches das Profil der Stadt völlig veränderte. In diesem Bereich mangelt es heutzutage circa 100 Millionen Bürgerinnen und Bürgern in den meisten Gemeinden Brasiliens immer noch an adäquaten Grundbedingungen - die Diskussion ist hochaktuell im Land. ${ }^{6}$ Die Weitsicht von Neves da Fontoura, auch seine Fähigkeit, sich intelligent anzupassen, die eigene kritische Meinung zum Ausdruck zu bringen, in die Opposition zu gehen, gelegentlich auch ins Exil, dafür aber im Dialog mit der Macht zu bleiben, sich mit den vorher kritisierten Machtinhabern zu versöhnen und aktiv an der entsprechenden Regierung teilzunehmen, zeugen von der Einzigartigkeit seiner Person in einem Land, dessen Geschichte sich durch instabile und konfliktbeladene Machtstrukturen charakterisiert.

In Hinblick auf die Bedeutung der sozialen, politischen und kulturellen Realität in von den Zentren weit entfernten Städten Brasiliens - wie Cachoeira do Sul ist kennzeichnend, dass Guimarães Rosa seine Lobrede auf Neves da Fontoura gerade mit Informationen über die eigene Geburtsstadt, das ebenso kleine und entlegene Cordisburgo, beginnt und mit dieser Erwähnung abschließt. Ebenso kommt nun in diesem Text wiederholte Male die Verbindung zwischen einer Person und dem Namen ihrer jeweiligen Geburtsstadt als Hypokoristikum («hipocorístico») vor. Es wird berichtet, dass bei der Arbeit die Kollegen diese Anreden verwendeten: nicht nur werden João Neves «Cachoeira» und Rosa «Cordisburgo» (oder aus Versehen nach der benachbarten Stadt «Barbacena») genannt, auch den Arbeitskolle-

5 Die Anspielung auf die guten Werke in Cachoeira do Sul kommen bezeichnenderweise vor im Zusammenhang eines Gesprächs über die Vereinbarkeit vom Leben als Politiker und dem Leben als Künstler: «Uma vez, por exemplo, descansávamos, especulando disso e daquilo, chegou-se a confronto entre o político e o artista. Precipitei-me a grado de argumentos e exercício. Neves, repartido absorto, externou-se então em frases muito planas, não dissertava, recordava. Falou das obras que pudera promover na Cachoeira, de tanto que no Brasil precisava de urgente ser feito, imaginava humildes enormes realizações.» Aus: «Discurso de posse».

6 Siehe dazu: https://www12.senado.leg.br/noticias/materias/2019/09/25/brasil-tem-48-da-populacao-sem-coleta-de-esgoto-diz-instituto-trata-brasil. Letzter Zugriff: 30.1.2018. 
gen Rui Ribeiro Couto nennt man «Pouso Alto». Der Text räumt jedenfalls dem formalen Umgang mit Eigennamen eine große Bedeutung ein. Zum Beispiel wird der Name des Gelobten mit einer goldenen Quelle («Fons Aurea, fonte áuria, Fontoura») in Verbindung gebracht sowie mit der Klarheit des weißen Schnees («alvo Neves - em nitidez»). Die Lenkung der Aufmerksamkeit auf die Sprachform und symbolische Bedeutung der Namen hebt die Einsicht der Figuren in die Ambivalenz ihrer eigenen Rolle in der Politik hervor: sie wirkten diskret, peripher, unprätentiös, waren «provinzieller» Herkunft, doch ihre Wirkung wies große Kraft auf, eine Kraft, die aus dem Willen der Bevölkerung im Inneren des Landes entsteht. «João Neves», behauptet Rosa in seiner Rede, «firma residir no interior e na província ainda «a força do Brasil, especialmente nos maiores Estados»». Rosa stimmt dem «Ministro» zu, würdigt mit ihm die Provinz, die Kraft der kleineren Städte und der territorial größeren Bundesstaaten, die in der Bundespolitik einen allzu geringen Einfluss ausübten. Rosa unterstützt außerdem Fontouras Kritik an den Metropolen, die «zum Dilettantismus, zur Oberflächlichkeit, zum Epikureismus führen». ${ }^{7}$ Sie erwarten ihre Erneuerung durch die Aktivität von Vertretern entfernter Regionen, die die Erwartungen und den Willen der allgemeinen Bevölkerung beachten und im gesellschaftlichen Leben zur Geltung bringen. Letzten Endes sahen sich sowohl Neves da Fontoura als auch Rosa in der Politik als Akteure bei einem Prozess, den Jahre später Ángel Rama nicht zuletzt in Anbetracht des literarischen Werks vom Letzteren «narrative Transkulturation» nennen wird. ${ }^{8}$

Natürlich war Rosa sich dessen bewusst, dass seine Lage und die des Ministers doch auch durch eine weitere Ambivalenz gekennzeichnet waren: hoch positionierte Diplomaten sind keine Provinzler mehr, auch wenn sie Werte und Anschauungen aus der Provinz weiter pflegen wollen. Die internationale Mobilität im Auftrag des brasilianischen Staates macht aus ihnen Weltbürger. Beide gestalten durch ihre Tätigkeit die Entwicklung der Gesellschaft in Brasilien und die Entwicklung einer internationalen Gemeinschaft mit, zu deren Entstehung und Konsolidierung auch Brasilien, seine Großstädte, doch ebenso die provinziellen und ländlichen Regionen im Land einen Beitrag leisten sollten. Was könnte aber fürs Leben und Werk von Guimarães Rosa charakteristischer sein als die gewollte und förderliche Koexistenz zwischen spezifischen und universellen Inhalten und Formen? Er taucht in die eigene, literarisch umgestaltete Umgebung des biographischen Ursprungs ein mit dem Zweck, jede entlegene Region als Bühne menschlicher Dramen und Potenziale zu präsentieren: $O$ sertão é o mundo. In der Tat:

7 Vgl. «Discurso de posse». Rosa zitiert Neves da Fontoura, ohne die jeweilige Quelle zu erwähnen: «João Neves [...] imputa [...] às metrópoles levarem «ao diletantismo, à superficialidade, ao epicurismo»».

8 Ángel Rama: Transculturación narrativa en América Latina. Buenos Aires: El Andariego 2007. 
die multikulturelle und konfliktreiche Gesellschaft Brasiliens bat schon damals ein paradigmatisches Beispiel für die damals entstehenden Strukturen einer globalen Welt, in der es gilt, die Gleichzeitigkeit des Ungleichzeitigen zu beachten, ${ }^{9}$ und für die es von entscheidender Bedeutung ist, den dauerhaften Erhalt der Demokratie durch die Arbeit der Diplomatie zu stärken. ${ }^{10}$

In jedem Detail, insbesondere beim letzten öffentlichen Auftritt inszenierte nun João Guimarães Rosa unauffällig, aber konsequent seine Rolle als öffentliche Persönlichkeit - als Staatsmann und Schriftsteller zugleich. In seiner letzten Rede fungierten die eigene Biographie, doch vor allem die von João Neves da Fontoura als Sinnbild fürs Schicksal und den Auftrag Brasiliens in der Zeit des großen Kampfes zwischen Totalitarismus und Demokratie, die sich um den zweiten Weltkrieg ereignete. «No que refiro, sub-refiro-me.» - schreibt Rosa. «Não para a seus ombros aprontar minha biografia, isto é, retocar minha caricatura. Não eu, mas mim. Inábil redutor, secundarum partium, comparsa, mera pessoa de alusão, e há de haver que necessária.» ${ }^{11}$

Der Autor von Grande sertão: veredas (1956) war ein sorgfältiger Beobachter der Debatten und Entwicklungen in der brasilianischen Gesellschaft, ein literarischer Mitgestalter ihrer Inhalte und Formen im gespannten Netz sozialer Beziehungen, denen die friktionale ${ }^{12}$ Welt des literarischen Sertão besondere Ausdrucksweise und Sinngehalt bereitstellt.

\section{Zweiter Schritt: tief ins Gedächtnis schauen, der Gewalt auf der Spur}

A mim, a quem o conceito da soberania do povo suscitava ainda visos meu tanto teóricos, ensinou-me [João Neves] que ela tem outrossim carne e canseiras, tarimba e pão, consolação; mas, principalmente, certeza criadora.

\footnotetext{
9 Siehe u.v.a. mit Hinblick auf die Begriffsgeschichte Hanns-Georg Brose: Das Gleichzeitige ist ungleichzeitig. Über den Umgang mit einer Paradoxie und die Transformation der Zeit. In: HansGeorg Soeffner (Hg.): Unsichere Zeiten. Herausforderungen gesellschaftlicher Transformationen. Wiesbaden: VS Verlag 2008, S. 547-562.

10 Eine erste Studie zum Thema, vor allem aber eine Sammlung von Sachberichten Rosas als Diplomat präsentierte Heloisa Vilhena de Araújo: Guimarães Rosa: diplomata. Brasília, Ministério das Relações Exteriores: Fundação Alexandre de Gusmão 1987.

11 Aus: «Discurso de posse».

12 Vgl. Ottmar Ette: ZwischenWeltenSchreiben. Literaturen ohne festen Wohnsitz (ÜberLebenswissen II). Berlin: Kadmos 2005, S. 160f.
} 
Mit der Abschaffung der Sklaverei 1888 und der Erklärung der Republik ein Jahr später, der damit verbundenen Intensivierung der Einwanderung sowie dem exponentiellen Wachstum der Bevölkerung (beinahe Verdoppelung zwischen 1872 und 1900 auf 17,2 Mio. Einwohner, danach Anstieg auf 30,6 Mio. im Jahr 1920) geriet Brasilien in der Zwischenkriegszeit in die Lage einer ehemaligen Sklavenhaltergesellschaft, die brutal, rückständig und wirtschaftlich schwunglos gewesen war und sich auf einmal unter internem und externem Druck befand, zu einer modernen Industrienation zu werden.

Die Kaffeemetropole São Paulo war im Land größter Anziehungspunkt für innere und auswärtige Migration. Insbesondere dort, aber auch in Bundesstaaten wie Rio Grande do Sul und Minas Gerais, gab es zu Beginn des 20. Jahrhunderts erste moderne demokratische Bürgerinitiativen und soziale Bewegungen, die aber aufgrund dem baldigen Ausbruch der Diktatur im Jahr 1937 zur Kurzlebigkeit verurteilt waren. Auch in anderen Zentren entstand Schritt für Schritt ein urbanes fortschrittliches Leben, beispielsweise eine liberale Presse, die nicht nur auf Portugiesisch zirkulierte, sondern auch in den Sprachen der Gemeinden von Immigranten, die verhältnismäßig höhere Alphabetisierungsquoten aufwiesen. Doch die entscheidend erneuernde Kraft, die sich durchsetzen konnte, kam aus dem Militär - genauso wie zur Zeit der Erklärung der Republik im Jahr 1889. Gerade die Tenentes, die Leutnants, haben am Ende der 20er Jahre die politische Szene in Brasilien am stärksten bestimmt. In dieser bewegten Zeit begann Brasilien mit der Revolução de 30 seinen langen, ambivalenten und bei Weitem noch nicht abgeschlossenen Weg in die Moderne. Es war im Rückblick auf diese Vergangenheit und im Hinblick auf deren mögliche Folgen für die unmittelbare Zukunft des Landes, dass Bürger und Intellektuelle wie Neves da Fontoura und Guimarães Rosa handelten, kommunizierten und schrieben.

Mittlerweile wurde Grande sertão: veredas, das wohl berühmteste Werk des Romanciers aus Minas Gerais, ${ }^{13}$ in seiner sozialgeschichtlichen und politischen Reichweite adäquat wahrgenommen. ${ }^{14}$ Der Sertão, das Gebiet, aus dem der Ro-

13 Auch durch Adaptionen für Kino und als Fernsehserie gewann der Roman an Popularität. Siehe dazu u. a. Osvando J. de Morais: Grande sertão: veredas, o romance transformado: semiótica da construção do roteiro televisivo. São Paulo: Edusp/Fapesp 2000. 2019 war die Publikation einer neuen Ausgabe des Romans durch den Verlag Companhia das Letras eins der wichtigsten literarischen Ereignisse des Jahres in Brasilien.

14 Siehe dazu u.a. Heloisa Starling: Lembranças do Brasil: teoria política, história e ficção em 〈Grande sertão: veredas〉. Rio de Janeiro: Revan 1999. Willi Bolle: grandesertão.br. São Paulo: Editora 34/Duas Cidades 2004. Der letzt genannte Autor setzt den Roman mit Recht Texten wie Die Wurzeln Brasiliens von Sérgio Buarque de Holanda oder Herrenhaus und Sklavenhütte von Gilberto Freyre gleich. Eine bis dahin ausführliche Bibliographie zu João Guimarães Rosa findet man in: Cadernos de Literatura Brasileira 20-21 (2006), S. 283-342. Verfügbar unter https://issuu.com/ 
mancier stammt, ist eine zwischen den Bundesstaaten Minas Gerais, Goiás und Bahia gelegene, wenig fruchtbare Region im Zentrum Brasiliens. Von der Kolonialzeit bis hin ins frühe 20. Jahrhundert erhielt sich dort die Viehzucht als Hauptaktivität: Die Tiere lebten meistens frei und wurden von Züchtern und Händlern von Gegend zu Gegend getrieben. Die Bewohner des Sertão, die Sertanejos, lebten weit entfernt von der urbanen Welt, die gesellschaftliche Ordnung im Sertão war sehr stark von der Einteilung in Großgrundbesitzer und Landarbeiter geprägt, wobei sich noch starke Spuren der kolonialen Feudalherrschaft und Sklaverei fanden (und bis heute noch finden). Ein großer Teil der Bevölkerung des Sertão bestand jedoch auch in der Vergangenheit weder aus Herren noch aus Sklaven. Es ging hier um Menschen, die von Gelegenheitsarbeiten auf verschiedenen Landgütern lebten. Nicht selten bildeten sie eine Art von privatem Heer im Dienst eines Großgrundbesitzers; dann wurden sie Jagunços genannt. In der Geschichte Brasiliens kam es mehrmals dazu, dass Jagunços unabhängige Banden bildeten und gegen Staat und Landbesitzer revoltierten.

Grande sertão: veredas erzählt die Geschichte des ehemaligen Jagunço Riobaldo, der sich aus den Kämpfen im brasilianischen Sertão zurückzieht und als älterer Mann seine früheren Abenteuer berichtet. Auf seinem Bauernhof teilt Riobaldo einem Besucher die Erinnerungen an die vergangene Zeit mit. Das weite Spektrum an Gattungen unter dem Dach eines Abenteuerromans, innovative Formungen des Sprachduktus von Riobaldo, Protagonisten und rückblickendem Erzähler im Text, verbale Neuschöpfungen und Verweise auf Geschichte und soziale Gegebenheiten, all das dient der Besprechung von Fragen wie Liebe, Schuld, Rechtfertigung und Sühne aus dem Blickwinkel der Hauptfigur: Eine Zuneigung für den Jagunço Diadorim war der Grund, weshalb Riobaldo sich in seiner Jugend entschied, sich dessen Bande anzuschließen. Den Jungen Diadorim hatte Riobaldo kennengelernt, als beide noch Kinder waren und gemeinsam mit dem Boot einen Fluss überquerten. Die einmalige Begegnung prägte jahrelang das Gedächtnis und Gefühl Riobaldos, bis er Diadorim durch Zufall wieder begegnete. Das Entzücken der Kindheit wurde im Laufe gemeinsamer Abenteuer zu einem von homoerotischen Zügen geprägten Gefühl. Mit dieser zwar platonischen, aber auf jeden Fall verbotenen Liebe für Diadorim konnte Riobaldo nicht fertig werden. Die Freunde führten mit ihrer Bande den Krieg gegen Hermógenes, einen ehema-

ims_instituto_moreira_salles/docs/clb_guimar_es_rosa. Die Universität São Paulo (USP) unterhält seit einigen Jahren eine entsprechende digitale Bibliographie mit zurzeit über 5.600 Einträgen: https://www.usp.br/bibliografia/inicial.php?s=grosa. Das Archiv des Autors befindet sich im Instituto de Estudos Brasileiros (IEB) an dieser Universität. Seine Bibliothek wurde erstmals von Suzi Sperber bibliographiert und verdient weiterhin einer ausführlichen Erforschung. Dazu: Suzi Frankl Sperber: Caos e Cosmos: leituras de Guimarães Rosa. São Paulo: Duas Cidades 1976. 
ligen Kameraden, der den großen Chef Joca Ramiro getötet hatte. Nach einem angeblichen Pakt mit dem Teufel wurde Riobaldo zum Führer der Bande. Unter seiner Führung sollte die Gruppe die endgültige Schlacht austragen.

Erst als Diadorim Hermógenes tötete und im Kampf fiel, erfuhr Riobaldo, dass der begehrte Freund in der Tat eine Frau war, und zwar die Tochter Joca Ramiros, die seit ihrer Kindheit für das Kriegsleben eines Mannes im Sertão erzogen wurde.

Nach einer Zeit der tiefen Erschütterung empfing Riobaldo den Besuch seiner künftigen Ehefrau Otacília, die ihm von ihrer Familie zur Verlobten gegeben wurde. Kurz danach bekam er die Nachricht vom Tode seines Patenonkels Selorico Mendes, der ihn als Erben anerkannt und im Testament mit seinen zwei größten Landgütern bedacht hatte. So wurde Riobaldo in sehr kurzer Zeit zu einem sozial gut etablierten Großgrundbesitzer. Grande sertão: veredas ist daher auch ein Roman des sozialen Aufstiegs, eine konsequente Reflexion über Macht- und Arbeitsverhältnisse in der sich wandelnden brasilianischen Gesellschaft im Fin-de-Siècle.

Die Figur eines Deutschen, Vupes, spielt im Werk eine zwar sekundäre und episodische Rolle, nach der inneren Logik des Werks ist er aber unentbehrlich für dessen vollständige Erschließung. So heißt es im Roman: «Vupes! Wenn ich den auslasse, wird meine Geschichte unvollständig.» ${ }^{15}$ Die Aktivität des Händlers aus Deutschland besteht darin, den Grundbesitzern Werkzeuge, Schädlingsbekämpfungsmittel und einfache Maschinen wie "Windmühlen zum Wasserpumpen» ${ }^{16}$ anzuliefern und zu verkaufen. Der alte Riobaldo habe gehört, Vupes «sei noch am Leben und wohne heute begütert in der Hauptstadt, sei Besitzer eines großen Handels- und Ladengeschäfts, mit dem er sein Geld verdient hat». ${ }^{17}$ Er sei «ein ganzer Kerl. Ein anständiges Haus. Ein systematischer Kopf». ${ }^{18}$ Und - ganz wichtig im Kontext unseres Arguments - «trotz des heillosen Durcheinanders von Politik und Krawall in der Gegend ließ der sich nicht aus der Fassung bringen.» ${ }^{19}$ Mit

15 Zitiert nach der deutschen Ausgabe João Guimarães Rosa: Grande sertão. Übers. von Curt Meyer-Clason. Berlin/Weimar: Aufbau 1969, S. 81. Im portugiesischen Original: «Ah, eh e não, alto-lá comigo, que assim falseio, o mesmo é. Pois ia me esquecendo: o Vupes! Não digo o que digo, se o do Vupes não orço - que teve, tãomente.» Aus: João Guimarães Rosa: Ficção completa. 2 Bd. Rio de Janeiro: Nova Aguilar 1994. Hier Bd. 1, S. 50. Alle weiteren Zitate (nur mit Seitenangabe, falls abweichend) ebenso.

16 Rosa: Grande Sertão, ebda.; Portugiesisch: «[...] papa-vento, desses moinhos-de-vento de sungar água [...]» (ebda.).

17 Ebda.; Portugiesisch: «Diz-se que vive até hoje, mas abastado, na capital - e que é dono de venda grande, loja, conforme prosperou» (ebda.).

18 Ebda.; Portugiesisch: «[...] indivíduo, mesmo. Pessoa boa. Homem sistemático [...]» (ebda.).

19 Ebda.; «[...] com toda a confusão de política e brigas, por aí, e ele não somava com nenhuma coisa $[. .] ».($ ebda.) 
Vupes reist ein europäischer Fremder durch die weit entlegene Region in der Provinz. Riobaldo fällt auf, dass der Deutsche «geregelt und genau [lebte] und nie seinen kühlen Kopf [verlor]». ${ }^{20}$ Bezeichnend sei in Vupes dessen Fähigkeit, «trotz Sertão [...] stets seine Bequemlichkeit zu finden». ${ }^{21}$ Das heißt, die Tätigkeit von Vupes, im Beruflichen aber auch im Privaten, verändert seine Umgebung, er lässt sich nicht von äußeren Gegebenheiten einschüchtern, gestaltet seine Umwelt und führt neue kulturelle und materielle Elemente ein.

Im Text lenkt die Begegnung von Riobaldo und Vupes die Aufmerksamkeit des Lesers auf die sozioökonomische Situation der beiden Figuren. Bei der Einführung von Vupes in den Roman, die hier gerade besprochen wurde, ist Riobaldo bereits ein wichtiger Jagunço und verdient im Sertão Respekt. Auch darum ist Vupes erfreut, Riobaldo zu begegnen. Denn er habe erfahren, Riobaldo sei sehr tapfer, und Vupes müsse eben solch jemanden haben, der ihn auf der Reise begleiten würde: «vierzehn Tage, Sertão jetzt ganz durcheinander, wilde Leute, alles...». ${ }^{22}$ Riobaldo, der als tapferer Führer anerkennt wird, «schwoll der Kamm», und er war nun auf einmal «stolz auf [s] einen Beruf» des Jagunço. Er ist sich außerdem der Tatsache bewusst, dass, «wenn einer bei den Jagunços ist, er kein Auge für die Armut ringsum [hat], er sie nicht einmal sieht». ${ }^{23}$ Riobaldos Rolle ist ambivalent: zum einen ein tapferer Held und zum anderen selbst ein Bestandteil in der dämpfenden Repressionsmaschinerie der ungleichen und gewaltsamen Gesellschaft Brasiliens. Vupes will in die Stadt São Francisco reisen, angeblich ist das auch der Weg Riobaldos, und nach kurzer Verhandlung übernimmt Letzterer in Begleitung seines Kumpels Sesfrêdo, eines weiteren Gliedes in der stark hierarchisierten Ordnung des Sertão, die Führung.

In diesem Zusammenhang erwähnt Riobaldo eine vorige Begegnung mit dem Ausländer, die ein paar Seiten später im labyrinthischen Erzählen des Romans ausführlich präsentiert wird. Damals, wenig Jahre früher, war es mit Vupes ganz anders gewesen. Riobaldo hatte gerade das Haus seiner Kindheit empört verlassen, nachdem es ihm klar wurde, dass sein Patenonkel Selorico Mendes in der Tat sein natürlicher Vater war. Riobaldo hatte Hab und Gut gesammelt und sich in den benachbarten Ort Curralinho begeben, um sich von Leuten zu verabschieden, die in seiner Kindheit und Pubertät wichtig gewesen waren. Er hatte vor, nach dem Aufenthalt in Curralinho zur Jagunço-Lebensführung anzusetzen. Im Dorf

20 Ebda., S. 83; «O Vupes vivia o regulado miúdo, e para tudo tinha sangue frio» (ebda., S. 51).

21 Ebda.; Portugiesisch: «[...] Assim no sertão, e ele formava conforto, o que queria» (ebda.).

22 Ebda., S. 82; Portugiesisch: «quinze dias, sertão agora aqui muito atrapalhado, gente braba, tudo...» (ebda., S. 50).

23 Ebda.; Portugiesisch: «Um jagunceando, nem vê, nem repara na pobreza de todos, cisco» (ebda.). 
lebte zum Beispiel sein Lehrer, Mestre Lukas. Außerdem zwei Mädchen, Miosótis und Rosa'uarda, die er als seine «Schwärme betrachtet hatte» ${ }^{24}$, und von denen er sich verabschieden möchte. Daher besucht er unter anderem den Vater von Rosa'uarda, den «Türke[n] und Kaufmann» Assis Wababa (Rosa 1969: 140), Freund seines Patenonkels. Bei Wababa war eben der Lieferant Vupes zu Besuch. Bei Tisch, an dem der junge Riobaldo teilnehmen durfte, erzählte nun der Deutsche dem (eigentlich libanesischen) Kunden Erfreuliches über das wirtschaftliche Wachstum der Region, etwa den baldigen Ausbau der Bahnlinie bis nach Curralinho, wo der Gastgeber sein Geschäft betrieb.

Diese Neuigkeit wird auch von Riobaldo begeistert aufgenommen. Als Erzähler erinnert er sich zurück: «Ich ließ meiner Phantasie freien Lauf und bildete mir ein, durch den Fortschritt der Neuzeit würden auch meine Probleme endgültig gelöst werden. Ich sah mich bereits wohlbestallt und steinreich. Ich sah sogar, wie es gut wäre, wenn es Wahrheit würde.» ${ }^{25}$ Die Stimmung war so gut, dass Riobaldo sich in solche Träumereien verlor und ihn der Gedanke durchfuhr, er könnte «seine Lage damit retten», dass er bei Vupes «als Reisender arbeitete und Werkzeuge und Baumwollentkernungsmaschinen verkaufte». Er, der Rosa'uarda sehr mochte, hatte gerade erfahren, dass sie «verlobt [war] und in wenigen Monaten Salino Cúri heiraten [sollte] - der wie ihr Vater Türke und Kaufmann war.» ${ }^{26}$ Die Nachricht über Ausbau der Bahnlinie; den Traum, dadurch steinreich zu werden; und der Plan, als Weg in die wirtschaftliche Etablierung erstmals für Vupes zu arbeiten, hängen in der Episode zusammen. Riobaldo wollte sich möglichst schnell wirtschaftlich etablieren, vielleicht träumte er noch davon, Rosa'uarda zu seiner Frau zu machen, und fragte nun Vupes, ohne lange zu überlegen, ob dieser ihn als Verkäufer nicht anstellen wollte. Auf der Stelle bekam er ein «Nix!» als Antwort, und ihm wurde auf einmal klar, dass «ein Mensch, der als Freund höflich ist, als Vorgesetzter ein Raubein und ein Nörgler werden kann». ${ }^{27}$ Das Zusammensein mit den Herren wurde ihm peinlich, er fühlte sich minderwertig, verabschiedete sich und ging fort. Die Episode wird mit einem emblematischen Gedicht ab-

24 Ebda., S. 82; Portugiesisch: «[...] as que, no Curralinho, eu pensava que tinham sido minhas namoradas» (ebda., S. 51).

25 Ebda., S. 140; Portugiesisch: «[...] eu entrei no que imaginei - na ilusãozinha de que para mim também estava tudo assim resolvido, o progresso moderno: e que eu me representava ali rico, estabelecido» (ebda., S. 84).

26 Ebda.; Portugiesisch: «[...] a Rosa’uarda agora estava sendo noiva, para se casar com um Salino Cúri, outro turco negociante [...]» (ebda.).

27 Ebda., S. 141; Portugiesisch: «mesmo pessoa amiga e cortês, virando patrão da gente, vira mais rude e reprovante» (ebda., S. 84). 
geschlossen, nachdem Riobaldo ein letztes Mal an Rosa'uarda denkt und ihm deutlich wird, dass er sie für immer verloren hatte:

Hätte dein Vater Geld,

Einen Handel dazu,

Ich nähm dich im $\mathrm{Nu}$,

Und mein wär die Welt... ${ }^{28}$

Dem jungen Mann wird die eigene sozioökonomische Lage bewusst, und er entscheidet sich nun, sein eigenes Leben zu beginnen, «um mehr [zu] lernen und [sich] städtische Manieren anzueignen ${ }^{29}$, eben das, was er bei Vupes im Laufe des ganzen Romans so bewunderte.

Durch die Korrelation der beiden Episoden wird klar, dass bei der sozialen und ökonomischen Integration Riobaldos in die Gesellschaft Vupes eine sinnbildliche Rolle spielt, die für den Umgang mit Urbanität, Wirtschaft, Handel und Arbeitswelt steht. Nachdem Riobaldo Großgrundbesitzer geworden ist, erscheint der Name des Deutschen ein letztes Mal, kurz vor dem Ende des Romans: Vupes läuft Riobaldo über den Weg, ${ }^{30}$ als dieser zu Quelemém geht, einem anderen Großgrundbesitzer, der zu Riobaldos geistigem Berater geworden war, nachdem dieser das Landgut seines Paten übernommen hatte.

Vupes war apropos auch dem Zuhörer des alten Riobaldo bekannt, einem Bewohner der Stadt, der im Sertão auf Reisen war und als Gast bei seinem Gesprächspartner Unterkunft fand. Als der ehemalige Jagunço dies erfuhr, fragte er den Gast: «Sie haben ihn gekannt? Die Welt ist doch ein Dorf! Wie sprechen Sie den Namen aus? Wusp? Ganz recht. Seu Emilio Wuspes... Wúpsis... Vuspes. Also dieser Vupes tauchte dort auf und erkannte mich, weil er mich von Curralinho her kannte.» ${ }^{31}$ Auffällig ist hierbei, wie in der Antrittsrede im Jahre 1967, das Wortspiel mit dem Namen des Fremden. Und nun: In der Bibliothek Guimarães Rosas findet sich ein Band mit der berühmten Arbeit über die deutsche Immigration in Brasilien A aculturação dos alemães no Brasil (1946), dessen Autor zwar nicht wie der deutsche Händler im Roman Emilio Wuspes oder Wúpsis heißt, dafür aber Emilio Willems: derselbe Vorname, ein ähnlicher Nachname. Gewiss verweist die

28 Ebda.; Portugiesisch: «Seu pai fosse rico, / tivesse negócio, / eu casava contigo / e o prazer era nosso...» (ebda.).

29 Ebda., S. 142; Portugiesisch: «a fito de desenvolver mais estudos e apuramento só de cidade» (ebda.).

30 Ebda., S. 661.

31 Ebda., S. 81; Portugiesisch: «Ah, o senhor conheceu ele? Ô titiquinha de mundo! E como é mesmo que o senhor fraseia? Wusp? É. Seo Emílio Wuspes... Wupsis... Vupses. Pois esse Vupes apareceu lá, logo vai me reconheceu, como me conhecia do Curralinho» (ebda., S. 50). 
Figur von Vupes auf die aufmerksame Lektüre der Abhandlung von Willems, bei der sich Rosa theoretische Kategorien und empirische Daten aneignen konnte, die später in seinem Roman Anwendung fanden. Das Exemplar enthält Anmerkungen gerade auf den Seiten, wo auf die Bedeutung der Veränderungen in der sogenannten «materiellen Kultur» ${ }^{32}$ verwiesen wird. Die Verfügbarkeit von Werkzeugen, Häusern, Maschinen, Waffen, der soziale Gebrauch der Sprache, die Heiratsregeln und die Mitgiftverwaltung in den Dörfern und auf den Landgütern, die soziale Ausgrenzung oder Integrierung je nach Herkunft und sozialer Rolle, das sind die Themen, die Rosa im Buch von Willems hervorhebt und studiert, um sie später in seiner literarischen Produktion programmatisch darzustellen.

«Mein ganzes Leben hat mich das Fremdländische angezogen», ${ }^{33}$ das behauptet Riobaldo im Zusammenhang seiner Zuneigung zu Rosa'uarda. Zehn Seiten später wiederholt sich das Argument, wenn er sich entscheidet, nach dem besagten Verlassen des Hauses seiner Kindheit Assis Wababa zu besuchen: «Ich wollte [...] zu Seo Assis Wababa - im Augenblick konnte ich nur Fremde ertragen, mir Fremde, Fremdländische!» ${ }^{34}$ Bei Wababas wird Arabisch gesprochen, «Uarda» heißt in dieser Sprache «Blume», was im Roman nicht erklärt wird, doch für aufmerksame Leser durch die befremdende Schreibweise des Namens Rosa'uarda (Setzung eines halben Anführungszeichen zwischen «Rosa» und «uarda») klar angedeutet wird.

Die genaue Berücksichtigung einer Durchdringung der brasilianischen Gesellschaft durch internationale Elemente, die sie transformieren und mitgestalten, weist auf die große Bedeutung, die Guimarães Rosa diesen Elementen sowohl im politischen Wirken als auch im literarischen Schaffen einräumt. Wie in seiner Rede zum Antritt in die Unsterblichkeit des literarischen Ruhms spielt die Internationalität und deren Bedeutung im Kampf um eine möglichst gerechte Gesellschaft in Brasilien und in der Weltgemeinschaft eine entscheidende Rolle.

32 Emilio Willems: A aculturação dos alemães no Brasil. Estudo antropológico dos alemães e seus descendentes no Brasil. São Paulo: Companhia Editora Nacional 1946.

33 Rosa: Grande sertão, S. 129; Portugiesisch: «Toda a vida gostei demais de estrangeiro» (Rosa: Ficção completa, Bd. 1, S. 71).

34 Ebda., S. 138; Portugiesisch: «aquela hora eu queria só gente estranha, muito estrangeira, estrangeira inteira!» (ebda., S. 83). 


\section{Dritter Schritt: Brasilien vor der Weltgemeinschaft}

João Neves da Fontoura wurde von Guimarães Rosa im November 1967 als einer seiner politischen Meister präsentiert. Nicht nur in der Politik, sondern auch in der Diplomatie. Er lobt sowohl seine Aktivität als Botschafter in Portugal unter Salazar als auch seine Tätigkeit als Außerminister in zwei verschiedenen Amtsperioden.

Für João Guimarães Rosa begann die eigene diplomatische Laufbahn im Ausland, als er gerade nach Deutschland unter Naziherrschaft entsandt wurde, um das Amt des Vizekonsuls in Hamburg durchzuführen. In der hanseatischen Stadt verbrachte er die Zeit von 1938-1942, wurde zum Zeugen der nationalsozialistischen Diktatur und der antisemitischen Geschehnisse in Dritten Reich. Doch auch mit dem Antisemitismus im eigenen Land musste er sich auseinandersetzen, einem unbeleuchteten Kapitel der brasilianischen Geschichte, das man erst zu Beginn des neuen Jahrtausends angefangen hat zu bewältigen. ${ }^{35}$

Die Kurzgeschichte «A velha» («Die Greisin») sei hier als paradigmatisches Beispiel erwähnt und dessen Inhalt mit den Worten von Aline Alves-Bergerhoff kurz dargelegt:

[Im Text] versucht eine alte deutsche Frau, Dame Verônika, in der Anwesenheit des Ich-Erzählers, eines brasilianischen Diplomaten in Hamburg, die jüdischen Wurzeln ihrer Tochter, Dame Angélika, auszulöschen, die sie vom verstorbenen Ehemann der Dame Verônika geerbt habe. Dies macht die verzweifelte Mutter, indem sie ein jahrelang bewahrtes Geheimnis ihrer eigenen Biographie verrät, nämlich, dass die Geburt ihrer Tochter das Ergebnis einer in Brasilien erlebten Liebesaffäre zwischen ihr und einem brasilianischen Mann gewesen sei. Sie begleitete zu der Zeit ihren Ehemann in Brasilien, der als Hebräisch-Lehrer des Kaisers wirkte. Dame Verônika stellt sich vor dem brasilianischen Diplomaten bloß in der Hoffnung, ein Ausreisevisum für ihre Tochter zu erhalten. Denn als Halbjüdin, die sie in der Tat nicht sei, sei sie in Lebensgefahr. ${ }^{36}$

Im Erzähltext bespricht Guimarães Rosa auf subtile Art die problematische Position Brasiliens gegenüber der Frage des Antisemitismus vor und während dem

35 Siehe dazu insbesondere die Arbeiten von Maria Luiza Tucci Carneiro: $O$ anti-semitismo na Era Vargas. São Paulo: Perspectiva 2001.

36 Aline Alves-Bergerhoff: Der Stellenwert eigenkultureller Literatur mit deutschem Bezug für den DaF-Unterricht im internationalen Kontext. Das Beispiel von Guimarães Rosa in Brasilien. In: Jahrbuch Deutsch als Fremdsprache. Intercultural German Studies 42 (2019), S. $179 f$. 
Zweiten Weltkrieg. ${ }^{37}$ Es geht hier um die konsequente literarische Erzeugung eines archivalisch belegbaren Prozesses von Fehltritt und Schuldbekenntnis in Bezug auf die geheim gehaltene antisemitische Politik des Landes, mit dem sich erst heute die Diplomatie und die Gesellschaft Brasiliens konfrontiert sehen. Einzelwörter, kalkulierte Ausdrücke - Realitätsvokabeln könnte man sie mit Hermann Broch nennen -, die auch sinnlich-konkrete Hinweise auf Archivmaterial sind, berichten von der politischen Reifung eines Autors, der aber zu seiner Zeit nicht imstande war, gegen die offizielle Politik aufzutreten, und sie eher gehorsam erfüllt hat. Philologisch konsequent registriert Guimarães Rosa im literarischen Schreibprozess die Wegmarkierung zur Wiederaufnahme des Problems, die man erst Jahrzehnte später diskret aufbringen kann. Da geht es um eine Art von Wissen, von Lebenswissen, das allein philologisch und zugleich archivalisch wiedergewonnen werden kann. Am Anfang dieser Ausführungen kam das Argument zum Ausdruck, dass Guimarães Rosa seine Rolle als öffentliche Persönlichkeit inszenierte - als Staatsmann und Schriftsteller zugleich. Die eigene Biographie fungiere demnach als Sinnbild für Brasiliens Weg während des großen Kampfes zwischen Totalitarismus und Demokratie, die sich um den zweiten Weltkrieg ereignete.

Wenn ich hier Ottmar Ette in seinem Werk ZwischenWeltenSchreiben paraphrasieren darf, überlagern sich in dem Fall die Geschichten der Figur eines Diplomaten in der Fiktion und des rückblickenden diplomatischen Schriftstellers. Die Geschichten überlagern sich, und sie überlagern sich zugleich in ihrer jeweiligen Bedingtheit durch Geschichte. Wir sollten, laut Ettes Gedankengang, das erzählende Ich, das eine textinterne Instanz darstellt, mit dem realen, textexternen

37 Eine wichtige Dimension für die Lektüre der Texte von Guimarães Rosa sowie der Texte von seinem Übersetzer und Schriftstellerkollegen Curt Meyer-Clason ist die Betrachtung intellektueller Konstellationen (vgl. hierzu Martin Mulsow/Marcelo Stamm: Konstellationsforschung. Frankfurt/M.: Suhrkamp 2005), an denen sich beide Denker zwischen den 1920er und den 1960er Jahren beteiligten, d.h. die Berücksichtigung und Situierung verschiedener Akteure in einem Assoziationsnetzwerk von Intellektuellen, Schriftstellern und Literaturkritikern, von Diplomaten und Kulturmittlern wie João Neves da Fontoura, aber auch von anderen Akteuren wie dem brasilianischen Diplomaten Mario Calabria ( $\left.{ }^{\star} 1923\right)$ und der Ehefrau von Guimarães Rosa, Aracy Moebius de Carvalho (1908-2011). Aracys Leben, die wie ihr Ehemann in den Jahren 1938-42 einer Tätigkeit am brasilianischen Konsulat in Hamburg nachging, wurde vor wenigen Jahren in Brasilien ausführlich aufgearbeitet, nicht zuletzt im Hinblick auf ihr Engagement für bedrohte Juden in Deutschland. Ihre Biographie trägt den Titel Justa (Gerechte) in Anspielung auf den Ehrentitel der Gerechten unter den Völkern, den ihr (als einzigem weiteren brasilianischen Bürger außer dem Botschafter Souza Dantas) der Staat Israel im Jahre 1982 verlieh. Mônica Raisa Schpun: Justa. Aracy de Carvalho e o resgate de judeus: trocando a Alemanha nazista pelo Brasil. Rio de Janeiro: Civilização Brasileira 2011. 
Autor nicht gleichsetzen oder verwechseln. Der Autor als Gewährsmann des von ihm Erzählten ist in jedem Fall eine Figur, die im Gegensatz zum realen Schriftsteller textintern bleibt. Die autobiographische Anspielung in «A velha» ist aber im Falle Guimarães Rosas so evident wie notwendig, um die Leser, die textintern selbstverständlich ebenfalls Leserfiguren sind, mit den realen textexternen Lesern zu verknüpfen, welche den literarischen Text auf der Suche nach einer aktuellen Lesergemeinschaft erst schaffen muß. Das Autobiographische ist funktional von größter Wichtigkeit, bedingt aber zugleich die Form des Textes. Es ist emotional ergreifend, ja empörend, und schreibtechnisch subtil, doch letztlich nicht die Bedeutungsebene, von der aus alle anderen Elemente der Erzählung Sinn erzeugen. Der Sinn, der aber erzeugt wird, weist außer Zweifel auf das kodierte Autobiographische hin. Und es wird somit zum Bestandteil einer philologischen Materialität und Wirksamkeit des Textes - insbesondere im heutigen brasilianischen Prozess der Vergangenheitsbewältigung, auch in Bezug auf die diktatorische Vargas-Ära. Von großem Interesse für die tiefgreifende Analyse des Textes ist daher die Hervorhebung von konsequenten Wortspielen, die allein im Übersetzungsprozess vom Portugiesischen ins Deutsche und umgekehrt bemerkbar sind. Auf formaler Ebene operiert Rosa mit verschiedenen sprachlichen Eigenschaften des Deutschen bzw. des Paares Deutsch-Portugiesisch. Die im Portugiesischen befremdende, aber dadurch auch besonders schöne Formulierung «embaixada-dejó» bezeugt zum Beispiel den unmittelbaren Gebrauch des deutschen Wortes «Hiobsbotschaft». Zu Beginn der Kurzgeschichte wird die Situation von Juden, die in Hamburg nach Hilfe suchen, folgendermaßen beschrieben: «[...] o Consulado invadindo-se de judeus, sob mó de angústias, famintos de partir, sofridos intenso, em desengano, público pranto e longo estremecer, quase cada rosto prometendose a coativa esperança final do suicídio.» ${ }^{38}$ Auch das Flehen von Frau Wetterhuse bzw. Dame Veronika nach Hilfe erreicht in diesem Kontext das Konsulat: «[...] seguinte, na semana, voltava, a súplica, embaixada-de-jó, apelo insistido.» 39

Durch die wörtliche, frei-kreative Wiedergabe von «Hiobsbotschaft» ins Portugiesische als «embaixada-de-jó» spielt Rosa mit der Doppelbedeutung von «Botschaft» in der deutschen Sprache, wo das Wort einmal «Mitteilung» (port.

38 João Guimarães Rosa: Ave, palavra. Rio de Janerio: José Olympio 1970, S. 108. In deutscher Übersetzung (von Anna Scheifler; überarb. von Judith Jennert und Paulo Soethe, unveröffentl.): «Das Konsulat wurde von Juden überrannt, die, zermahlen von Angst, nach der Ausreise hungerten, von lang anhaltendem Schaudern erfüllt und getäuscht, ihr Leid öffentlich zur Schau stellten, und auf jedem Gesicht sah man die Erwartung, dass als letzte Hoffnung nur der Selbstmord blieb.»

39 Ebda. Im deutschen Wortlaut: «in der Woche darauf, erneut die Bitte, das Flehen, Hiobsbotschaft, die Forderung beharrlich.» 
«notícia»), einmal «Auslandsvertretung» (port. «embaixada») meinen kann. Im Portugiesischen gibt es dennoch die Koinzidenz nicht, dass eine einzige Vokabel beide Bedeutungen meint, so ist man notwendigerweise auf das Wortspiel im translingualen Ort zwischen Deutsch und Portugiesisch angewiesen, wenn man im portugiesischen Original das Wort in seiner Komplexität verstehen will. Im Kontext der Kurzgeschichte wird dadurch die Ambiguität der Vokabel hervorgehoben, um die Nähe zwischen der deutschen und der brasilianischen Art und Weise mit der Situation umzugehen, zu verdeutlichen. Das Leiden des biblischen Juden Hiob, Grund dafür, dass «Hiobsbotschaft» eine schlechte Nachricht meint, findet in der brasilianischen Auslandsvertretung, die der alten Dame im Text ein Ausreisevisum verweigert, Erfüllung. Allein im Kontext der Transformation vom Deutschen ins Portugiesische und des Portugiesischen durch das Deutsche bringt das Wort den erzielten Sinn.

In ähnlicher Hinsicht hebt ein anderer Übersetzungsprozess die sinnlichakustische Dimension der Sprache hervor, indem aus der geläufigen, aber akustisch speziell klingenden Vokabel «Hitlergreueltaten» Guimarães Rosa den suggestiven Neologismus «hitlerocidades» schafft, eine Mischung aus dem Namen Hitler und dem portugiesischen Wort für Greueltaten «atrocidades»: «Só então entrou a falar sob a força de fatos: dos campos-de-prisão, as hitlerocidades, as trágicas técnicas, o ódio abismático, os judeus trateados.» ${ }^{40}$

Die Erfahrung von Krieg, Gewalt, Willkür, staatlich legitimierter Menschenverachtung und Rassismus hinterließ im politischen Bewusstsein und literarischen Werk Guimarães Rosas Spuren, die es immer wieder gilt hervorzuheben ${ }^{41}$

40 Deutsch: «Erst jetzt begann sie von Tatsachen bedrängt zu sprechen: von den Konzentrationslagern, den Hitlergreueltaten, den tragischen Praktiken, dem haltlosen Hass, den gequälten Juden.»

41 In anderen Texten wies ich bereits auf diese Frage. Vgl. u. a. Paulo Soethe: Os humores de Wotan: fontes alemãs de Guimarães Rosa. In: Antonio Donizeti da Cruz/Lourdes Kaminski Alves: Literatura e sociedade no contexto latino-americano. Cascavel, PR: Editora da Unioeste, S. 223-240. Ebenso motivierte ich ehemalige Schüler und Schülerinnen, dass sie sich mit dem Thema befassen, beispielsweise mit Hinblick auf die Bedeutung des Themas für die Ausbildung von Deutschlehrern und -lehrerinnen in Brasilien. Siehe z.B. Maria Carolina Mocellin de Farias: Anderssprachige Literatur mit Deutschlandbezug und die Ausbildung von Deutschlehrern: Guimarães Rosas «Deutsche Erzählungen" in Brasilien. Masterarbeit - Bundesuniversität von Paraná/Universität Leipzig 2015. Verfügbar unter http://acervodigital.ufpr.br/handle/1884/41956. Aline Alves-Bergerhoff: Der Stellenwert eigenkultureller Literatur mit deutschem Bezug für den DaF-Unterricht im internationalen Kontext. Das Beispiel von Guimarães Rosa in Brasilien. In: Jahrbuch Deutsch als Fremdsprache 42 (2016), S. 177-193. Siehe außerdem zum Thema u. a.: Marcel Vejmelka: Guimarães Rosa na Alemanha: a metafísica enganosa. In: Scripta 5/10 (2002), S. 412-424; ders.: Kreuzwege: Querungen - João Guimarães Rosas ‘Grande sertão: veredas〉 und Thomas Manns 〈Doktor Faustus` im interkulturellen Vergleich. Berlin: Edition Tranvía 2005. Adriana Jacobsen/So- 
und im breiteren Kontext der Debatte über die Chancen der Demokratie - gestern und heute - in einem weltvertretend so ungleichen Land wie Brasilien in Betracht zu ziehen. Es geht letzten Endes darum, wie Ottmar Ette in Anlehnung an Roland Barthes schon formulierte, «durch die Rückgewinnung von Offenheit in unserer Sichtweise der Vergangenheit jene Freiheit wiederzugewinnen, die uns - vor dem Hintergrund einer historisch möglichst exakten Rekonstruktion - die Konstruktion einer Offenheit der Zukunft, ja einer Eröffnung von Zukünften erlaubt». ${ }^{42}$

raia Vilela: Diário de uma Busca. In: Humboldt 49/95 (2007), S. 74-75; dies.: Guimarães Rosa na Alemanha. In: Cadernos de Literatura Brasileira 20-21 (2006), Beilageheft; dies.: outro sertão (Dokumentarfilm, 2013, 73 Min.). Georg Otte: O «Diário Alemão〉 de João Guimarães Rosa - Relato de um projeto de pesquisa em andamento. In: Leila Parreira Duarte (Hg.): Veredas de Rosa II. Belo Horizonte: PUCMinas 2003, S. 285-290. Daniel Bonomo: A biblioteca alemã de João Guimarães Rosa. In: Pandaemonium germanicum 16 (2010), S. 155-183. Jaime Ginzburg: Notas sobre o «Diário de guerra de João Guimarães Rosa». In: Aletria 20/2 (2010a), S. 95-107; und ders.: Guimarães Rosa e o terror total. In: Elcio Cornelsen/Tom Burn (Hg.). Literatura e guerra. Belo Horizonte: Editora UFMG 2010b, S. 17-27. Reinaldo M. Marques: O diário de Guimarães Rosa na Alemanha: a escritura e a leitura como coleção. In: José Cirillo/Ângela Grando (Hg.). Processos de criação e interações: a crítica genética em debate nas artes, ensino e literatura. Belo Horizonte: C/Arte 2008, S. 294-302. 42 Ottmar Ette: LebensZeichen. Roland Barthes zur Einführung. Hamburg: Junius 2011, S. 15. 